\title{
Changes of Operative Performance of Pulse Pressure Variation As A Predictor of Fluid Responsiveness in a Swine Model of Endotoxin Shock
}

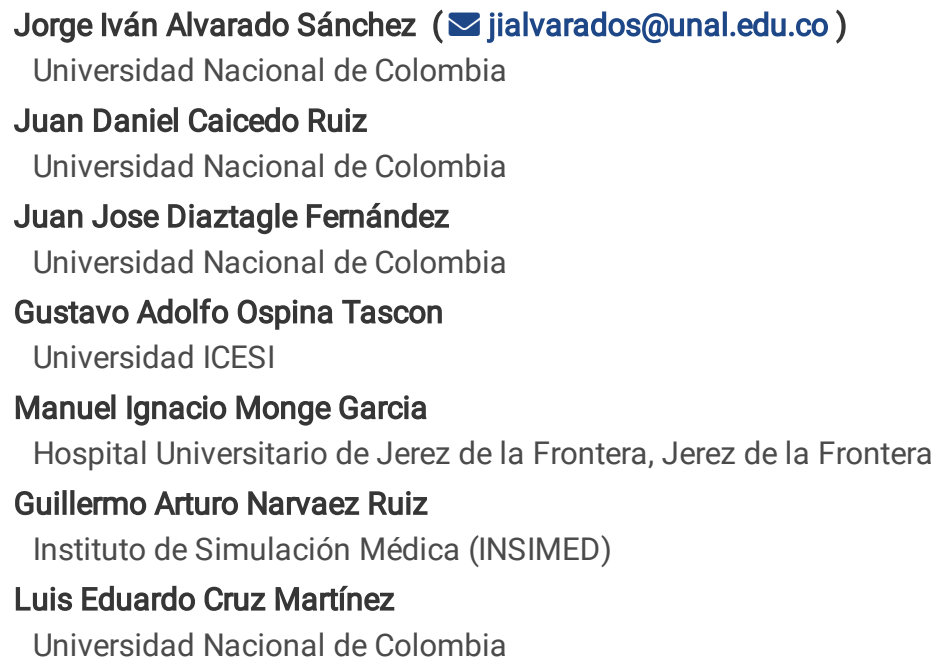

\section{Research Article}

Keywords: responsiveness, endotoxin, PPV, MAP

Posted Date: September 3rd, 2021

DOI: https://doi.org/10.21203/rs.3.rs-812479/v1

License: (c) (i) This work is licensed under a Creative Commons Attribution 4.0 International License. Read Full License 


\section{Abstract \\ Background}

Several limitations regarding pulse pressure variation (PPV) use have been reported. Our aim was to describe changes in the PPV operative performance as a predictor of fluid responsiveness during the development of a swine endotoxin shock model and to assess hemodynamic variables associated with PPV changes.

\section{Methods}

A swine porcine endotoxin shock model was established ( $E$. Coli 055:B5 endotoxin) in 7 pigs, and 3 pigs were included in the control group. The endotoxin was infused until the mean arterial pressure (MAP) dropped below $50 \mathrm{mmHg}$ (TH0); then, the model animal was reanimated with fluids and vasopressors. We performed fluid challenges every hour for 6 hours. ROC curve analysis was conducted. Additionally, a linear mixed model was performed.

\section{Results}

The area under the curve (AUC) of PPV decreased from $0.95(0.81-1.00)$ to $0.60(0.17-1.00)$ at TH0. Its cutoff increased from $10.5-22.00 \%$ at THO. PPV showed an inverse relationship with stroke volume, mean systemic filling pressure (MSFP), MAP, and systemic vascular resistance $(S V R)(p<0,001, A I C=111.85)$.

\section{Conclusions}

The PPV operative performance as a predictor of fluid responsiveness decreased with the progression of endotoxic shock. This result could be due to the inverse association with MAP and SVR.

\section{Introduction}

Appropriate fluid therapy is key for the management of critically ill patients in intensive care units or the operating room ${ }^{1-2}$. The use of intravenous fluid therapy based on physiological variables related to fluid responsiveness has been beneficial in these patients ${ }^{3-4}$, whereas its use without defined hemodynamic goals has been associated with an increased medical complication rate 5 .

Dynamic indices related to cardiac preload have been generally used as predictors of fluid responsiveness. A positive response to fluid challenge is usually defined as a $10-15 \%$ increase in cardiac output (CO) or stroke volume (SV) ${ }^{6}$. Among these indices, pulse pressure variation (PPV) has been used as a predictor of fluid responsiveness in mechanically ventilated patients in several clinical settings. However, some limitations have been described ${ }^{7-8}$.

Although this hemodynamic variable has been studied for 20 years, little is known about its operative performance as a predictor of fluid responsiveness during hypotensive states, such as septic shock. Moreover, Monge et al. showed that PPV is related to effective arterial elastance ${ }^{9}$, a variable that summarizes the features of arterial vascular load in humans ${ }^{10}$. Therefore, its operative performance and its cutoff as a predictor of fluid responsiveness could change in hypotensive states.

This study aimed to describe changes in the PPV operative performance and cutoff of PPV as a predictor of fluid responsiveness during the induction of endotoxic shock in a swine model. We also used a statistical model to explore the possible relationship between PPV and other hemodynamic variables that could explicate this decrease in the PPV operative performance.

\section{Materials And Methods}

\section{Protocol}

The study was carried out in accordance with the principles for the care and use of animals in research established by the Guide for the Care and Use of Laboratory Animals (NIH Guide) ${ }^{11}$, Resolution 008430 of 1993 issued by the Colombian Ministry of Health, and Law 84 of 1989 issued by the Congress of Colombia, the "Estatuto Nacional de Protección de Animales" (National Statute for the Protection of Animals). Additionally, this research was conducted according to the Animal Research: Reporting of In Vivo Experiments (ARRIVE) guidelines ${ }^{12}$. 
This study was approved by the Bioethics Committee of the Faculty of Veterinary Medicine of Universidad Nacional de Colombia (CB-FMVZUN-031-19). The study was conducted at the simulation laboratory of Instituto de Simulacion Medica (INSIMED) from January to December 2019. Ten female pigs (Yorkshire $40 \pm 1.0 \mathrm{~kg} ; \pm 4$ months old) were premedicated using tiletamine-zolazepam (Zoletilß, Virbac Colombia) at $4.4 \mathrm{mg} / \mathrm{kg}$ doses through intramuscular injection. Then, each pig was cannulated through the marginal ear vein, and anesthesia was induced through the inhalation of isoflurane at a 1.5 minimum alveolar concentration (MAC) using an anesthesia mask. Once under general anesthesia, the pig was intubated and mechanically ventilated with a tidal volume of $10 \mathrm{ml} / \mathrm{kg}$ and a respiratory rate of 20 breaths/minute. For the maintenance of general anesthesia, isoflurane at doses of 1.5 MAC was used. Additionally, in all animals, the internal jugular vein and the femoral artery were cannulated using a central venous catheter (ARROW® CV-17702) and an artery catheter (PiCCO® PV2015L20-

A). The quality of blood pressure signals was tested using a rapid washout test. All measurements were performed with the animal in a supine position, considering the phlebostatic axis as the zero reference.

Animals were placed on a stationary operating table and thermoregulated using medical blanket warmers, keeping their body temperature at a minimum level of $38^{\circ} \mathrm{C}$. During the development of the model, intravenous fluid was administered using normal saline solution (SS) at an infusion rate of $3 \mathrm{ml} / \mathrm{kg} / \mathrm{hour}$.

\section{Measurements}

A monitor with the pulse contour cardiac output system (TFT Mindray BeneVision N22 Patient Monitor) was used. CO was calculated as the average of three thermodilution boluses $\left(20 \mathrm{ml}\right.$ of $<8^{\circ} \mathrm{C} \mathrm{SS}$ through the jugular venous catheter). SV was calculated as $\mathrm{CO} /$ heart rate (HR). Systemic vascular resistance (SVR) and PPV were automatically calculated by the PiCCO® system. Finally, MSFP was estimated using the method of Parkin ${ }^{13}:$ MSFP $=0.96(C V P)+0.04(M A P)+0.5(C O)$

\section{Experimental protocol}

After hemodynamic stabilization (variation in MAP $<10 \%$ for at least 10 minutes), animals were assigned to the control group (3 animals) or the endotoxin group (7 animals).

Animals in the endotoxin group received a continuous infusion of endotoxin (LPS E. Coli 055: B5, Sigma, St. Louis, MO) through the central venous catheter at an infusion rate of $7 \mu \mathrm{g} / \mathrm{kg} / \mathrm{hour}$ that was increased every 10 minutes (7, 14, and $20 \mu \mathrm{g} / \mathrm{kg} / \mathrm{hour}) \mathrm{until}$ reaching 20 $\mu \mathrm{g} / \mathrm{kg} / \mathrm{hour}^{14}$. The infusion of endotoxin ended when an MAP $<50 \mathrm{~mm} \mathrm{Hg}$ for at least 10 minutes was achieved. Pigs in the control group were not administered endotoxin. Time zero (TO) was defined in the endotoxin group and the control group as the time immediately after hemodynamic stabilization.

When an MAP $<50 \mathrm{~mm} \mathrm{Hg}$ was reached in the endotoxin group (TH0), a fluid load was administered at a dose of $20 \mathrm{ml} / \mathrm{kg}$ for 20 to 30 minutes through the central venous catheter. In the control group, a similar fluid load was administered at 3 hours of observation (TH0). This time was selected in the control group because it was the median time required to reach an MAP $<50 \mathrm{~mm} \mathrm{Hg}$ for at least 10 minutes during the pre-experimental standardization phase of the endotoxin models. Then, a noradrenaline infusion at a dose of $0.05 \mathrm{mcg} / \mathrm{kg} / \mathrm{minute}$ was started after the fluid load, and the infusion rate was increased $0.05 \mathrm{mcg} / \mathrm{kg} /$ minute every 5 minutes until an MAP of $65 \mathrm{mmHg}$ was reached. Noradrenaline was not administered in the control group. After completing the protocol, all animals were euthanized by a certified veterinarian with a bolus of pentobarbital + diphenylhydantoin at $100 \mathrm{mg} / \mathrm{kg}$ (Euthanex®), in accordance with the international criteria for this procedure established by the American Veterinary Medical Association (AVMA).

\section{Fluid challenges}

A total of 6 fluid challenges were performed in each group. The first three fluid challenges were performed every hour starting at T0 (T0, T1, and T2). Afterward, three fluid challenges were performed every hour starting at TH0 (TH0, TH1, and TH2). Hemodynamic measurements were performed each time. This approach allowed us to assure that both groups had the same amount of fluid administered and that the variables could be compared.

All fluid challenges consisted of $4 \mathrm{ml} / \mathrm{kg}$ SS IV infused for 5 minutes through a central venous catheter. This is a standardized approach to perform a fluid challenge in humans ${ }^{15}$. Animals in which a fluid challenge induced an increase in $\mathrm{CO}>10 \%$ were defined as fluid responders 6 . CO was measured by transpulmonary thermodilution in all cases. The study protocol is depicted in Fig. 1.

\section{Statistical analysis}


Data are presented using medians and interquartile ranges. Receiver operating characteristic (ROC) curve analysis was conducted for each fluid challenge to assess changes in the PPV operative performance over time in the endotoxin group. The cutoff was calculated as the maximum (sum (sensitivity + specificity)).

Two-way analysis of variance (ANOVA) was conducted to assess differences between both groups. Additionally, one-way ANOVA was performed to determine changes in the variables over time in the endotoxin group. Post hoc analysis was carried out using the Bonferroni test.

A linear mixed model was performed to determine the associations between PPV and SVR, MAP, SV, baseline PPV, MSFP, and the endotoxin or the control group. Time was used as the random effect, the variables in the model were assessed by the restricted maximum likelihood (REML), and the contribution on each variable was quantified using the estimated value and its standard deviation. Models were compared using the Akaike information criterion (AIC), the Bayesian information criterion (BIC), and the REML. The models with the lowest AIC and BIC were considered the best models.

Data were analyzed using $\mathrm{R}$ statistical software. A p value $<0.05$ was considered statistically significant.

\section{Results}

An MAP $<50$ mmHg was reached between 2-3 hours after the start of endotoxin infusion. The changes in the hemodynamic variables are shown in Table 1 and Fig. 2. Systolic arterial pressure (SAP), diastolic arterial pressure (DAP), and MAP decreased, and their levels were lower in the endotoxin group. From T2 to TH1, SAP, DAP, and MAP were lower in the endotoxin group than in the control group ( $p<0.05)$ (Fig. 2C, $2 \mathrm{D}$, and $2 \mathrm{E}$, respectively). Additionally, in the endotoxin group, these variables decreased over time $(p<0.05)$. SVR showed similar behavior when measured at TH0 and TH1 ( $<$ 0.05) (Fig. 2F). The changes in the remaining variables are shown in Table 1. 
Table 1

Hemodynamic variables. Data are shown as median and interquartile range.

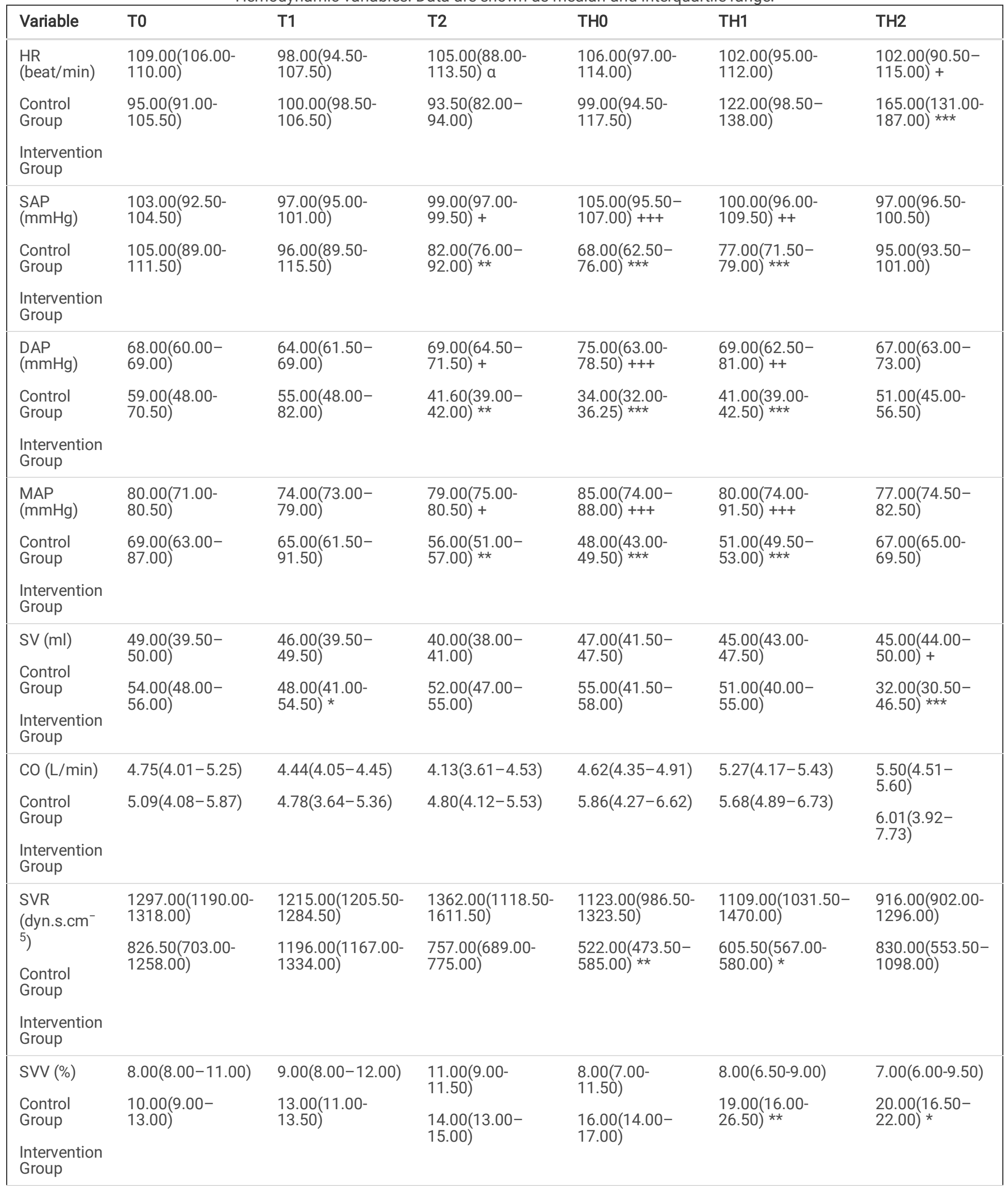




\begin{tabular}{|lllllll|}
\hline Variable & T0 & T1 & T2 & TH0 & TH1 & TH2 \\
\hline PPV (\%) & $10.00(8.50-$ & $9.00(9.00-12.50)$ & $7.00(7.00-11.00)$ & $9.00(7.00-$ & $9.00(6.50-11.00)$ & $7.00(6.50-$ \\
Control & $14.50)$ & $11.00(8.00-$ & $12.00(10.50-$ & $12.00(11.00-$ & $13.50)$ & $9.50)$ \\
Group & $15.00)$ & & $17.00)$ & $23.50(18.00-$ & $23.00)^{*}$ & $21.00(19.00-$ \\
Intervention & & & & & $24.00)^{*}$ \\
Group & & & & & \\
MSFP, & $16.88(14.69-$ & $16.90(16.76-$ & $18.84(17.37-$ & $16.92(16.45-$ & $19.33(18.90-$ & $18.95(17.18-$ \\
(mmHg) & $16.96)$ & $19.70)$ & $20.77)$ & $17.65)$ & $19.37)$ & $19.03)$ \\
$\begin{array}{l}\text { Control } \\
\text { Group }\end{array}$ & $16.03(15.52-$ & $16.86(14.45-$ & $14.94(14.88-$ & $15.42(13.16-$ & $17.37(17.03-$ & $14.99(13.16-$ \\
$\begin{array}{l}\text { Intervention } \\
\text { Group }\end{array}$ & $18.38)$ & $16.96)$ & $16.51)$ & $16.40)$ & $18.31)$ & $16.67)$ \\
\hline
\end{tabular}

$+=p<0.05,++=p<0.01,+++=p<0.001$ to comparison between Control and Intervention Group. ${ }^{*}=p<0.05, * \star=p<0.01, * * *=p<0.001$ to comparison between each time and the basal time on intervention group. CO, cardiac output; DAP, diastolic arterial pressure; HR, heart rate; MAP, mean arterial pressure; MSFP, mean systemic filling pressure; PPV, pulse pressure variation; SAP, systolic arterial pressure; SVR, systemic vascular resistance; SV, stroke volume; SVV, stroke volume variation.

\section{PPV operative performance in each fluid challenge}

The PPV was $11.0 \%(8.00-15.00 \%)$ at T0 and increased to $23.5 \%$ at THO (18.0-26.5\%) in the endotoxin group (Table 1, Fig. 2A).

A decrease in the PPV operative performance as a predictor of fluid responsiveness was observed, as the AUC decreased from 0.95 at T0 to 0.60 at THO. Moreover, its cutoff increased from $10.5 \%$ at T0 to $22.0 \%$ at THO (see Table 2). When noradrenaline infusion was initiated, the PPV operative performance increased to 0.75 at TH2.

Table 2

operative performance of PPV in each fluid responsiveness. AUC, area under curve.

\begin{tabular}{|llllll|}
\hline Fluid challenge & Sensibility (\%) & Specificity $(\%)$ & Threshold & AUC & Fluid responsiveness (\%) \\
\hline T0 & 100 & 80 & 10.5 & $0.95(0.81-1.00)$ & $28.6(p=0.25)$ \\
\hline T1 & 100 & 100 & 10.5 & $1.00(1.00-1.00)$ & $28.6(p=0.25)$ \\
\hline T2 & NA & NA & NA & NA & 0 \\
\hline TH0 & 100 & 60 & 22 & $0.60(0.17-1.00)$ & $28.6(p=0.25)$ \\
\hline TH1 & 100 & 83 & 23 & $0.70(0.26-1.00)$ & $14.3(p=0.05)$ \\
\hline TH2 & 100 & 60 & 22 & $0.75(0.35-1.00)$ & $28.6(p=0.25)$ \\
\hline
\end{tabular}

\section{Association between PPV and other hemodynamic variables}

The PPV model showed heteroscedasticity; therefore, it was necessary to transform PPV to the logarithm of PPV. The logarithm of PPV, which was obtained using a linear mixed model, showed a positive relationship with the baseline PPV; it was also higher in the endotoxin group. PPV showed an inverse relationship with SV, SVR, MAP, and MSFP. The analyses of all variables were statistically significant $(p<$ 0,0001) (Additional file 1, Table S1). The AIC was 111.85, the BIC was 139.12, and the REML was - 46.92. Additionally, the model showed homoscedasticity and normality of errors; therefore, its use was appropriate (Additional file 2, Figure S1).

The logarithm of PPV used in the present study was calculated as follows:

LogPPV $=4.26+1.73^{*} 10^{-2}$ (baseline PPV $)+0.39$ (endotoxin group) $-1.78 \times 10^{-2}(\mathrm{SV}(\mathrm{ml}))-7.46 * 10^{-3}(\mathrm{MAP}(\mathrm{mmHg}))-2.05 * 10^{-2}(\mathrm{MSFP}$ $(\mathrm{mmHg})-3.38 * 10^{-4}\left(\mathrm{SVR}\left(\right.\right.$ dyn.s.cm $\left.\left.{ }^{-5}\right)\right)$

\section{Discussion}

This study observed a decrease in the PPV operative performance and an increase in the cutoff as a predictor in the endotoxin group when an MAP $<50 \mathrm{mmHg}$ was achieved. Moreover, when noradrenaline infusion was initiated, the PPV operative performance improved. These 
These findings should be considered when a fluid challenge will be performed in critically ill patients with hypotensive states, and PPV will be used as a predictor of fluid responsiveness. Some studies have reported several limitations of PPV as a predictor of fluid responsiveness ${ }^{7-8}$; however, we did not find studies that reported changes in the PPV operative performance in critically ill patients with low arterial pressure. We suggest that these findings can be explained by the relationship among MSFP, SVR, stressed volume, and unstressed volume. MSFP is one of the determinants of venous return, and this is determined by the relationship between the stressed/unstressed volume (30\%/70\%) 16 and SVR. In clinical conditions when SVR is low, MSFP decreases ${ }^{17}$, and the relationship between the stressed/unstressed volume changes, leading to a clinical condition of relative hypovolemia. This clinical condition may not show a fluid response despite a high PPV value because the fluid load increases in the unstressed volume, preventing an increase in MCFP and hence an increase in venous return ${ }^{18}$. Therefore, a high PPV does not mean that there is a high likelihood of fluid response in this setting. Moreover, when MAP increased due to noradrenaline infusion, the operative performance improved, hence confirming the relationship between the PPV operative performance and SVR described above.

Our findings also showed an increase in the cutoff when an MAP $<50 \mathrm{mmHg}$ was reached. Several cutoffs have been reported in the literature in different clinical settings; however, we are not aware of studies that reported an increase in the cutoff in patients with low arterial pressure. This parameter should be taken into account since it increases the amount of fluid used for reanimation, increasing the risk of fluid overload.

Other findings from our study included an inverse relationship between PPV and load arterial variables, such as SVR and MAP. Some studies support this relationship. Monge et al. showed an association between PPV and effective arterial elastance ${ }^{9}$, a variable that summarizes पthe features of arterial vascular load in humans ${ }^{10}$. Other studies described a decrease in PPV after alpha-agonist infusion in experimental models of hemorrhagic shock, which could suggest a relationship between PPV and load arterial variables ${ }^{19-20}$. We suggest that this relationship could explain the low PPV operative performance when low arterial pressure was achieved.

Finally, we suggest that these findings should not be considered a limitation of PPV; instead, PPV should be considered a variable of preload dependency or a variable that allows us to recognize changes in the relationship of the stressed/unstressed volume, and the absence of changes related to a fluid challenge should alert us of the need for increased SVR. Thus, the risk of fluid overload will decrease.

The present study had some limitations. First, the evolution of PPV observed in our study may have been caused by increased vascular permeability, resulting in a decreased stressed blood volume, or by splenic red cell sequestration, as has been widely reported ${ }^{21-24}$. Second, we used the MSFP formula proposed by Parkin and Leaning ${ }^{13}$ instead of using an invasive measure of this variable. Nevertheless, this formula has yielded good results over time in swine models ${ }^{25}$. Moreover, the MSFP values found in the present study are similar to those reported in studies conducted in humans ${ }^{26}$. New studies are needed that allow the determination of the causes of the worsening of the PPV operative performance in this setting, assessing stressed/unstressed volume, MSFP, and venous return.

In conclusion, the PPV operative performance decreased markedly, and the cutoff increased when MAP was $<50$ mmHg. We also found an inverse association between PPV and load arterial variables, such MAP and SVR, that could be related to changes in the PPV operative performance.

\section{Declarations}

\section{Acknowledgments:}

To MINDRAY Colombia and BIMEDCO-GEMEDCO Colombia for the provision of the cardiac monitoring equipment and devices used in the study. Also, to Instituto de Simulación Médica (INSIMED) for allowing us to use their simulation laboratory for conducting the study.

\section{Author contributions:}

Design: all authors. Experimental protocol: JIAS, JDCR, JJDF, GARN, LECM. Acquisition of data: JIAS, JDCR. Statistical analysis: JIAS. Interpretation of data: all authors. Wrote the manuscript: all authors. All authors read and approved the final manuscript.

\section{Additional Information}

They received financial support by Universidad Nacional de Colombia and Fundación Universitaria de Ciencias de la Salud. Also, the author(s) declared the following potential conflicts of interest with respect to the research, authorship, and/or publication of this article: M. 
Ignacio Monge García is a consultant for Edwards Lifesciences.

\section{References}

1. Venn, R. et al. Randomized controlled trial to investigate influence of the Fluid challenge on duration of hospital stay and perioperative morbidity in patients with hip fractures. Br J Anaesth, 88 (1), 65-71 (2002).

2. Yogendran, S., Asokumar, B., Cheng, C. D. \& Chung, H. F. A Prospective Randomized Double-Blinded Study of the Effect of Intravenous Fluid Therapy on Adverse Outcomes on Outpatient Surgery. Anesth Analg, 80, 682-686 (1995).

3. Lee, J. et al. Association between fluid balance and survival in critically ill patients.Journal of Internal Medicine. 2014;1-10.

4. Sirvent, J-M., Ferri, C., Baró, A., Murcia, C. \& Lorencio, C. Fluid balance in sepsis and septic shock as a determining factor of mortality. Am J Emerg Med, 33 (2), 186-189 (2015).

5. Kelm, D. J. et al. Fluid overload in patients with severe sepsis and septic shock treated with early goal-directed therapy is associated with increased acute need for fluid-related medical interventions and hospital death. Shock, 43 (1), 68-73 (2015).

6. Alvarado Sánchez, J. I., Amaya Zúñiga, W. F. \& Monge García, M. I. Predictors to Intravenous Fluid Responsiveness. J Intensive Care Med, 33 (4), 227-240 (2018).

7. Alvarado Sánchez, J. I. et al. Predictors of fluid responsiveness in critically ill patients mechanically ventilated at low tidal volumes: systematic review and meta-analysis. Ann Intensive Care, 11, 28 (2021).

8. Teboul, J. L., Monnet, X., Chemla, D. \& Michard, F. Arterial pulse pressure variation with mechanical ventilation. Am J Respir Crit Care Med, 199 (1), 22-31 (2019).

9. Monge García, M. I. et al. Dynamic Arterial Elastance as a Ventriculo-Arterial Coupling Index: An Experimental Animal Study. Front Physiol, 11 (April), 1-16 (2020).

10. Kelly, R. P. et al. Effective arterial elastance as index of arterial vascular load in humans., 86 (2), $513-521$ (1992).

11. National Research Council (US) Committee for the Update of the Guide for the Care and Use of Laboratory Animals. Guide for the Care and Use of Laboratory Animals 8th edn (National Academies Press (US), Washington (DC), 2011).

12. Reynolds, P. et al. Shock supports the use of animal research reporting guidelines. Vol. 38, Shock. 2012. p. 1-3.

13. Parkin, W. G. \& Leaning, M. S. Therapeutic control of the circulation. J Clin Monit Comput, 22 (October), $391-400$ (2008).

14. Hatib, F., Jansen, J. R. C. \& Pinsky, M. R. Peripheral vascular decoupling in porcine endotoxic shock. J Appl Physiol, 111, 853-860 (2011).

15. Cecconi, M., Parsons, A. K. \& Rhodes, A. What is a fluid challenge? Curr Opin Crit Care, 17 (3), 290-295 (2011).

16. Gelman, S., Warner, D. S. \& Warner, M. A. Venous Function and Central Venous Pressure., 108 (4), $735-748$ (2008).

17. Persichini, R. et al. Effects of norepinephrine on mean systemic pressure and venous return in human septic shock. Crit Care Med, $\mathbf{4 0}$ (12), 3146-3153 (2012).

18. Gelman, S. \& Bigatello, L. The physiologic basis for goal-directed hemodynamic and fluid therapy: the pivotal role of the venous circulation. Can J Anesth [Internet], 65 (3), 294-308 (2018).

19. Nouira, S. et al. Effects of norepinephrine on static and dynamic preload indicators in experimental hemorrhagic shock. Crit Care Med, 33, 2339-2343 (2005).

20. Bouchacourt, J. P., Riva, J. A. \& Grignola, J. C. The increase of vasomotor tone avoids the ability of the dynamic preload indicators to estimate fluid responsiveness.BMC Anesthesiol. 2013;13.

21. Sakai, I., Ishihara, H., Iwakawa, T., Suzuki, A. \& Matsuki, A. Ratio of indocyanine green and glucose dilutions detects capillary protein leakage following endotoxin injection in dogs. Br J Anaesth, 81 (2), 193-197 (1998).

22. van Eijk, L. T. G. J. et al. Microvascular permeability during experimental human endotoxemia: an open intervention study. Crit Care, 9 (2), R157-64 (2005).

23. Sánchez, M. et al. Comparison of fluid compartments and fluid responsiveness in septic and non-septic patients. Anaesth Intensive Care, 39 (6), 1022-1029 (2011).

24. Chien, S. et al. Blood volume and its distribution in endotoxin shock. Am J Physiol, 210 (6), 1411-1418 (1966).

25. Werner-Moller, P., Sondergaard, S., Jakob, S. M., Takala, J. \& Berger, D. Effect of volume status on the estimation of mean systemic filling pressure.J Appl Physiol. 2019;1503-13.

26. Repessé, X. et al. Value and determinants of the mean systemic filling pressure in critically ill patients. Am J Physiol - Hear Circ Physiol, 309 (5), H1003-7 (2015). 
Figures

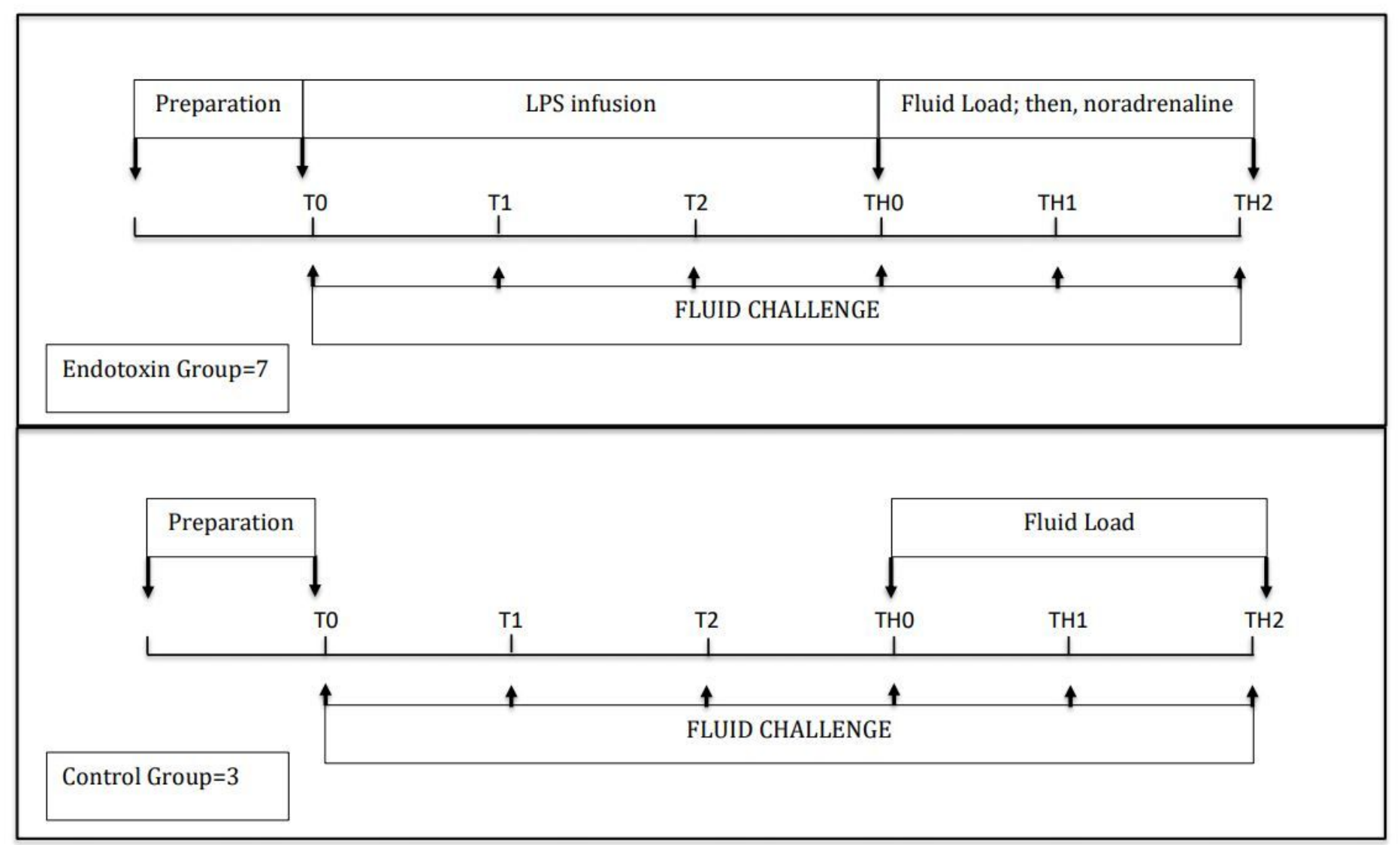

Figure 1

Study design. 

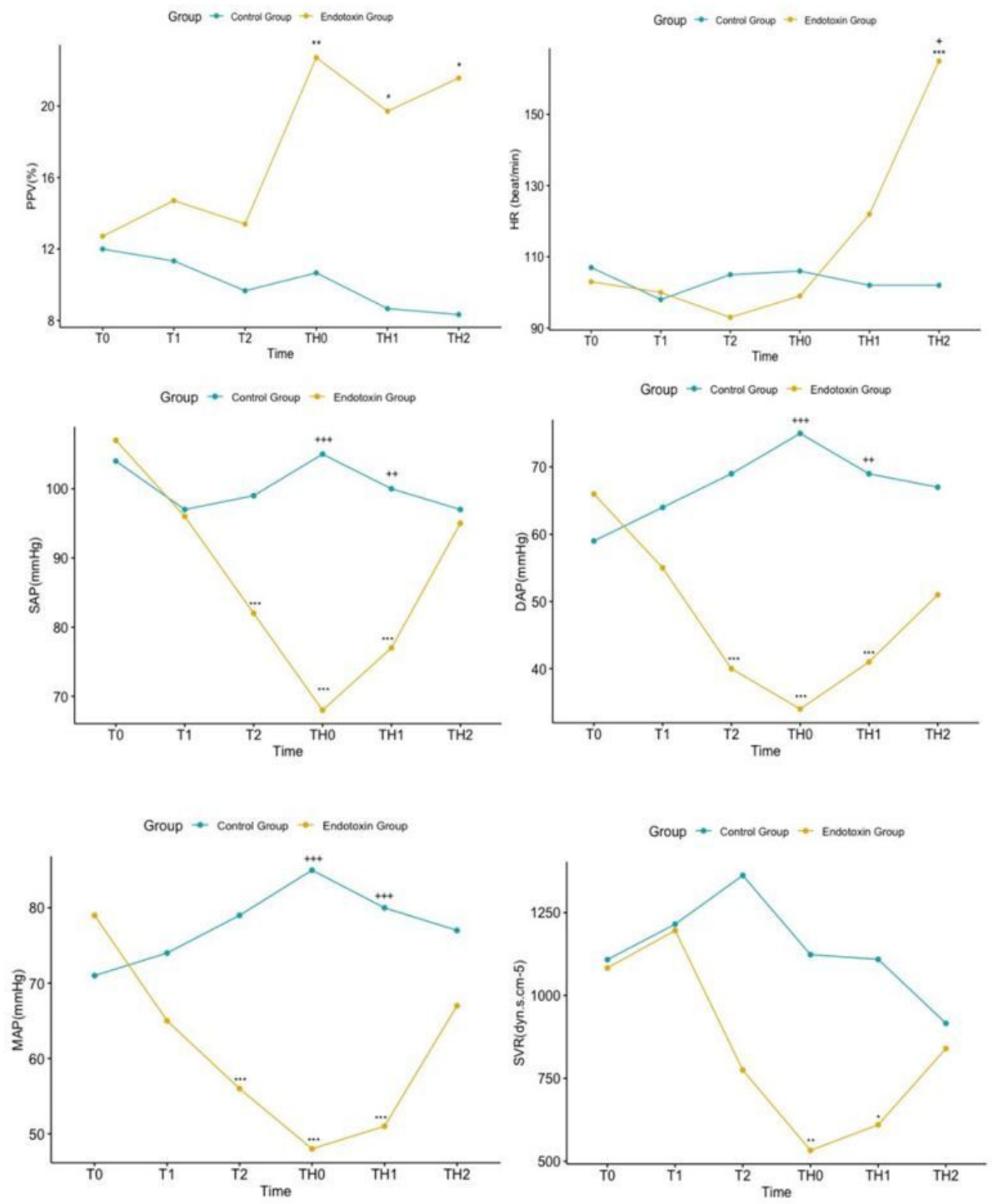

\section{Figure 2}

changes of hemodynamic variables for 6 hours of observation. A, changes of pulse pressure variation (PPV); B, changes of heart rate (HR); C, changes of systolic arterial pressure (PAS); D, changes of diastolic arterial pressure (DAP); E, changes of mean arterial pressure (MAP); $F$, changes of systemic vascular resistance (SVR ). $+=p<0.05,++=p<0.01,+++=p<0.001$ to comparison between Control and Endotoxin Group. ${ }^{*}=p<0.05, * *=p<0.01, * \star *=p<0.001$ to comparison between each time and T0 time on Endotoxin group.

\section{Supplementary Files}

This is a list of supplementary files associated with this preprint. Click to download.

- Additionalfile1tableS1.docx

- Additionalfile2figure1.pdf 